\title{
A Spatially Processed 3D Wideband Adaptive Conical Array System
}

\author{
Mohammad Ghavami \\ School of Engineering \\ London South Bank University \\ SE1 0AA, London, U.K. \\ Email: ghavamim@1sbu.ac.uk
}

\begin{abstract}
This paper presents a novel structure for an adaptive fully spatially processed wideband conical antenna array. A major advantage of this configuration is the frequency invariance of the directional patterns of the array within a relatively large fractional bandwidth which makes this array a potential candidate for wideband and ultra wideband (UWB) technology applications. Furthermore, unlike conventional wideband antenna arrays which use delay lines or time-domain filters, this system relies on fully spatial processing of the incoming or transmitted signals using a single real multiplier for each antenna element, without utilizing any phase shifters, digital filters or adjustable delays. Finally, due to the conical shape of the array configuration, beamforming in both azimuth and elevation angles is accomplished with symmetrical and uniform characteristics in all azimuth directions.
\end{abstract}

Index Terms-Wideband signals, Spatial processing, Array antenna, Conical arrays.

\section{INTRODUCTION}

Adaptive beamforming for the generation or detection of spatially directed signals can be used for various wideband applications such as audio, radar and UWB array signal processing. Very frequently, pure adjustable time delay elements have been widely used as a simple frequency dependent phase shifters in these applications. Frequency independence of delay arrays is maintained for the main direction of the beampattern, however the beamwidth and sidelobe attenuation level for different frequencies are not identical [1]. Tapped-delay-line and recursive filters [2][3] have also been employed on each branch of the array to allow each element to have a phase response that varies with frequency. Time domain wideband arrays involve a greater complexity of optimization algorithms for the desired pattern and they need very fast analog-to-digital converters for implementation of the filters.

A wideband smart antenna theory using rectangular array structures has been proposed in [4]. The main advantages of these structures are the frequency independence of the beam pattern over a large fractional bandwidth, and fully spatial signal processing which eliminates the requirements of using pure delay lines or the temporal processing of signals. On the other hands, these array systems suffer from non-uniform resolution of the directional patterns of the array over all azimuth angles. Moreover, they cannot handle elevation angles due to the two dimensional structure of rectangular arrays.
Frequency independent uniform and concentric circular arrays have been researched in the literature for beamforming and direction of arrival (DOA) estimation [5]. These arrays, like those similar to rectangular structures, cannot support elevation angles; however they demonstrate uniform characteristics for different azimuth angles.

In this study a three dimensional conical array structure with elements distributed over the surface of a cone is proposed. In the far field receiving mode, the signal received by each element is processed by a single real gain factor without requiring any phase shifter or delay element. This characteristic makes these arrays a good candidate for microstrip array antenna fabrication.

This paper is organized as follows. In Section II the structure of the conical array is explained. Section III investigates the adjustment process of the adaptive real multipliers allocated to each element. Some simulation results are presented in IV. Finally, section V concludes the paper.

\section{Adaptive Wideband Conical ARray}

Fig. 1 shows the basic structure of the proposed conical array configuration. Each antenna element is connected to an adaptive real multiplier $C_{m n}$. The resultant signals are then summated to generate the output signal. There are basically two general methods to calculate the multipliers of the conical arrays:

1) Optimization-based techniques: The desired array directional pattern is decided based on the desired angles of arrival, the undesired interference angles, and the dedicated frequency response of the UWB system. An optimization algorithm is used to synthesize the required pattern [6].

2) Adaptive array techniques: In this method, adjustable multipliers are computed iteratively using an adaptation technique which requires a training sequence as the desired signal [7].

The method used in this paper is the adaptive adjustment of the coefficients based on the directions of the desired and interference signals. Fig. 2 illustrates the locations of the antenna elements and the parameters used in the proposed structure. The array system consists of $M N$ elements which are located on a conical surface with a base angle $\beta$. The horizontal circles, centralized around the $z$ axis on and parallel to $x-y$ 


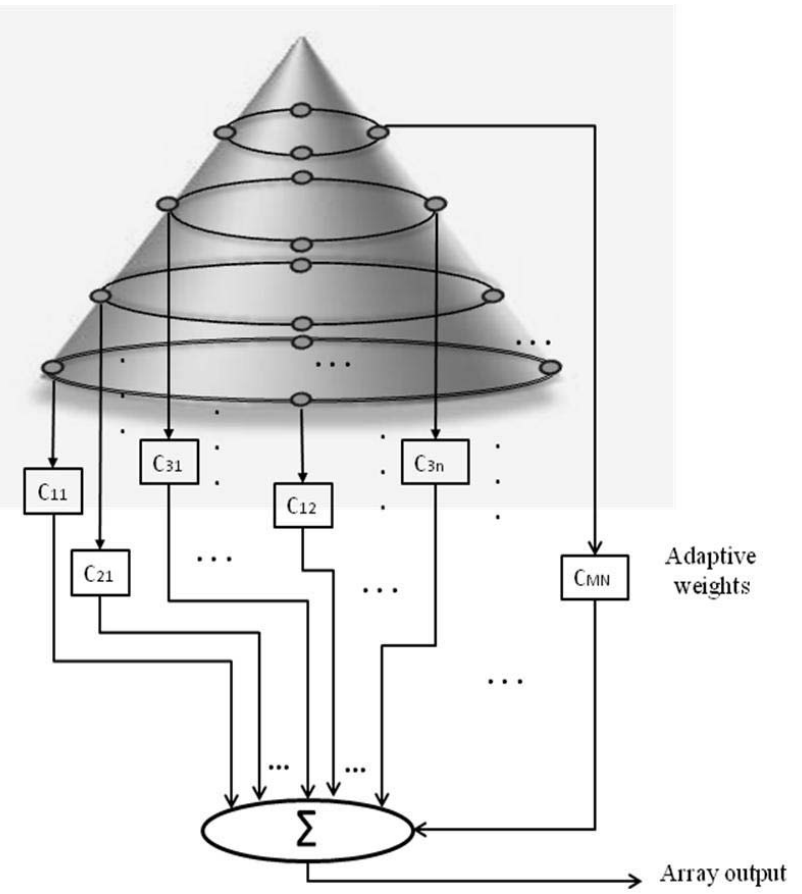

Fig. 1. Basic structure of an adaptive conical array. Each array element is connected to a real-valued weight $C_{m n}$.

plane are equally spaced and indexed by $m=1,2, \ldots, M$. On each circle there are $N$ antenna elements indexed by $n=0,1, \ldots, N-1$. The incline length of cone is taken as $M d$, with no element on the vertex. The length $d$ is defined as

$$
d=\frac{c}{f_{0 l}+f_{0 h}}
$$

where $c$ is the speed of light and $f_{0 l}$ and $f_{0 h}$ are the lowest and highest frequencies of operation, respectively. Equation (1) indicates that the distance along the incline of the cone between two antenna elements with the same index $n$ is half of the wavelength corresponding to the center frequency of the operational signals. This is the common choice for inter-element spacing of linear arrays. The angle $\alpha=\frac{2 \pi}{N}$ is a constant for all $M$ circles. The radius of each circle is represented by $r_{m}$ and is calculated from $r_{m}=r_{1} \frac{M-m+1}{M}$, where $r_{1}=M d \cos \beta$.

With reference to the geometry depicted in Fig. 2 the three dimensional coordinates of each $M N$ antenna element are written as $\left(x_{e}(m, n), y_{e}(m, n), z_{e}(m, n)\right)$, where calculations show that:

$$
\begin{aligned}
& x_{e}(m, n)=r_{m} \cos (n \alpha) \\
& y_{e}(m, n)=r_{m} \sin (n \alpha) \\
& z_{e}(m, n)=(m-1) d \sin (\beta)
\end{aligned}
$$

Assuming that the array system is used in receiver configuration, the incoming wave arrives to the array with the azimuth and elevation angles of $\theta_{0}$ and $\phi_{0}$, respectively. With reference to the origin, the incoming signal is arriving at each antenna

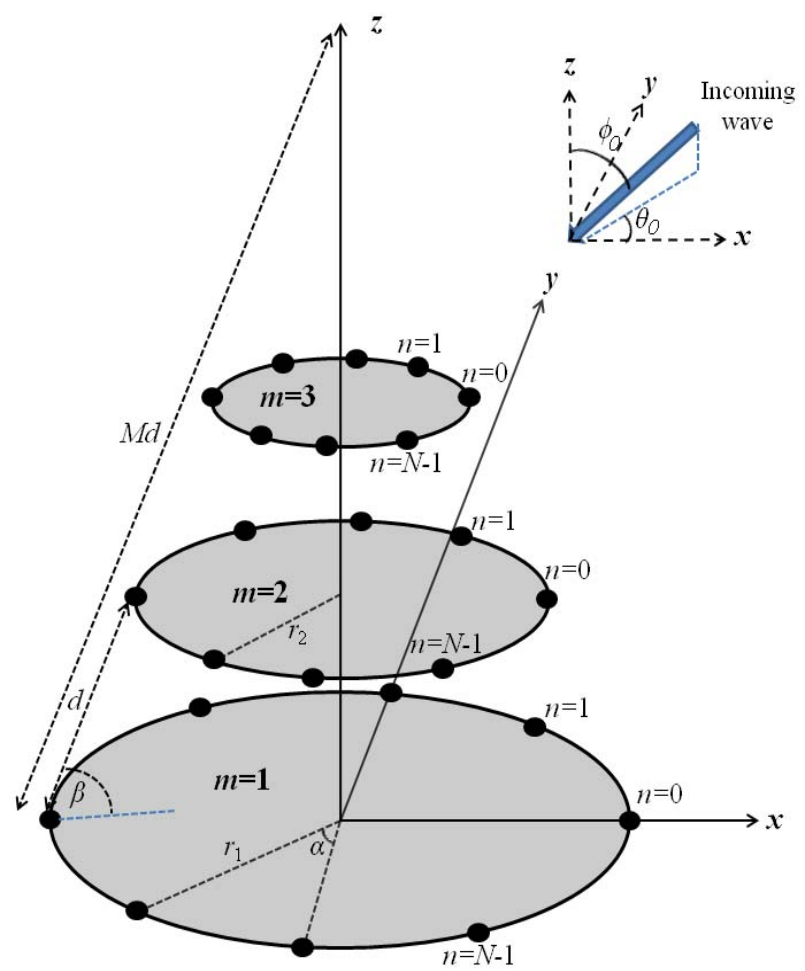

Fig. 2. A total of $M N$ antenna elements are located on the perimeters of parallel circular plates.

elements with a time delay that can be calculated from the following equation:

$$
\begin{aligned}
\tau_{0}(m, n)= & \frac{1}{c}\left[x_{e}(m, n) \cos \left(\theta_{0}\right) \sin \left(\phi_{0}\right)\right. \\
& +y_{e}(m, n) \sin \left(\theta_{0}\right) \sin \left(\phi_{0}\right) \\
& \left.+z_{e}(m, n) \cos \left(\phi_{0}\right)\right]
\end{aligned}
$$

In addition to the desired signal $s_{0}\left(t ; \theta_{0}, \phi_{0}\right)$, the system may also experience one or more interfering signals, $s_{i 1}\left(t ; \theta_{i 1}, \phi_{i 1}\right), s_{i 2}\left(t ; \theta_{i 2}, \phi_{i 2}\right), \ldots$, and a noise signal $v_{m n}(t)$ which are added at each element to generate the resultant signal at each antenna element. The interfering angles are defined by $\left(\theta_{i 1}, \phi_{i 1}\right),\left(\theta_{i 2}, \phi_{i 2}\right), \ldots$. The resultant signal at each element can be written as follows:

$$
\begin{aligned}
s_{m n}(t)= & s_{0}\left(t-\tau_{0}(m, n)\right)+v_{m n}(t)+s_{i 1}\left(t-\tau_{i 1}(m, n)\right) \\
& +s_{i 2}\left(t-\tau_{i 2}(m, n)\right)+\cdots
\end{aligned}
$$

where parameters $\tau_{i 1}(m, n)$ and $\tau_{i 2}(m, n), \ldots$ are the corresponding time delays of the interference signals and are calculated in a similar manner to the delays of the desired signal explained in (3).

The received signal at each array element is then multiplied by adjustable real multipliers $C_{m n}$ in order to calculate an 
TABLE I

LIST OF PARAMETERS USED FOR SIMULATION.

\begin{tabular}{|c|c|}
\hline Quantity & Corresponding values \\
\hline$M$ & 8 \\
$N$ & $\theta_{0}=20^{\circ}, \phi_{0}=70^{\circ}$ \\
Desired signal & $\theta_{i 1}=-50^{\circ}, \phi_{i 1}=80^{\circ}$ \\
Interference 1 & $\theta_{i 2}=0^{\circ}, \phi_{i 2}=50^{\circ}$ \\
Interference 2 & $2.7 \mathrm{~dB}$ \\
Signal to interference and noise ratio & From $4 \mathrm{GHz}$ to $5 \mathrm{GHz}$ \\
Bandwidth of all signals & $60^{\circ}$ \\
\hline
\end{tabular}

output signal as follows:

$$
y(t)=\sum_{m=1}^{M} \sum_{n=0}^{N-1} C_{m n} s_{m n}(t)
$$

\section{Adjustment of Real Multipliers}

The error signal is defined as:

$$
e(t)=s_{0}(t-\Delta)-y(t)
$$

where $\Delta$ is a constant time delay added to make the adaptive process causal. Then, the adjustable coefficients $C_{m n}$ are updated using the well known least mean square (LMS) algorithm [8] as follows:

$$
C_{m n}(t+\delta t)=C_{m n}(t)+\mu e(t) s_{m n}(t)
$$

to direct the mean squared error (MSE) toward zero, where the constant parameter $\mu$ is the step size or convergence factor and controls the convergence speed and stability of the calculations, and $\delta t$ is the time interval between two successive iterations.

Several parameters control the final MSE such as the number of elements, the power of the input signals and the angle and frequency characteristics of all desired and interfering signals.

\section{Simulation Results}

The parameters used in the simulation are listed in Table I. As shown in Fig. 3 adaptation of the real coefficients using LMS algorithm shows a smooth convergence to the optimal solution without difficulty when the convergence factor is chosen properly. Fig. 4 demonstrates the directional pattern of the whole array with the main direction toward the azimuth angle $\theta_{0}=20^{\circ}$ and the elevation angle $\phi_{0}=70^{\circ}$. We can also observe that two deep nulls exist close to the location of interference signals. This figure is plotted for the center frequency of the operation, i.e., $f=4.5 \mathrm{GHz}$.

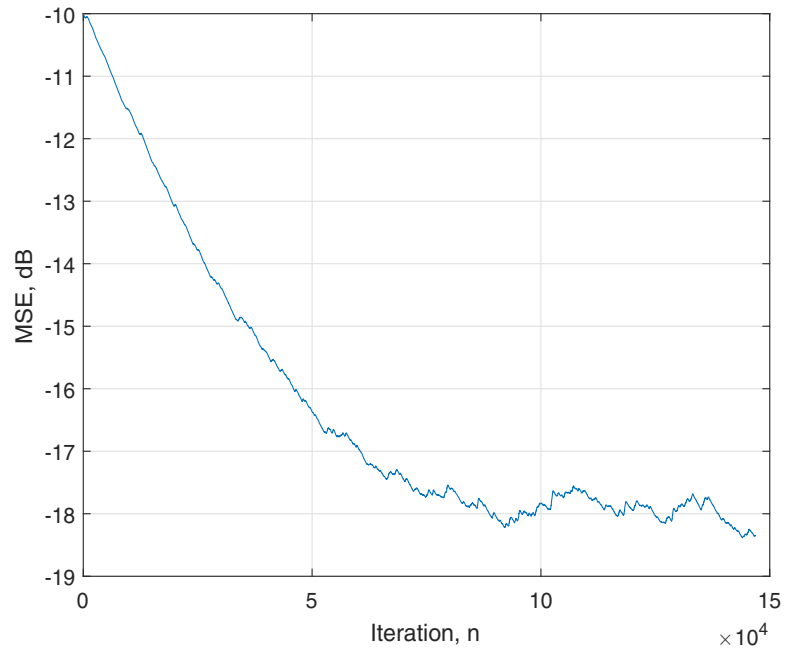

Fig. 3. MSE learning curve during the adaptation process.

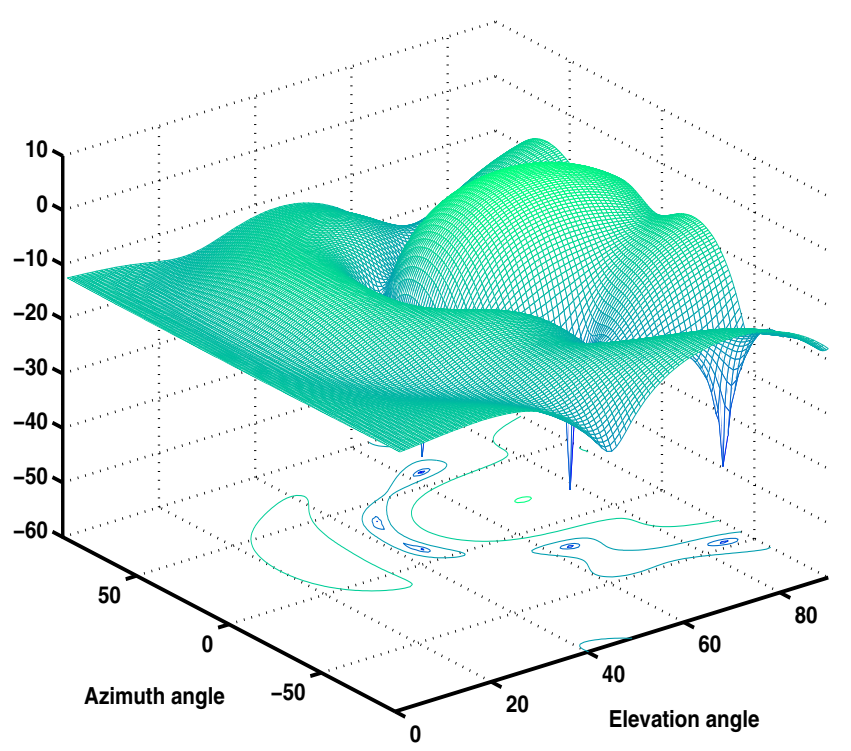

Fig. 4. Normalized directional pattern of the conical array (dB gain) for the center frequency of operation $f=4.5 \mathrm{GHz}$ clearly showing a global maximum point at the desired angles of azimuth and elevation and deep nulls near the angles of two interfering signals.

In order to investigate the performance of the array at other frequencies, Table II shows the obtained values for the attenuations of the interfering signals compared to the desired signal at three main frequencies. For example, at the center frequency $f=4.5 \mathrm{GHz}$, the attenuations are $27.1 \mathrm{~dB}$ and $18.2 \mathrm{~dB}$ for the interference signals 1 and 2, respectively.

Fig. 5 shows the variations of the beam pattern for the whole frequency range with respect to the azimuth angle $\theta$ when the elevation angle is kept at the desired value of $\phi_{0}=70^{\circ}$.

In a similar manner, Fig. 6 shows the variation of the directive pattern for the whole frequency range with respect 
TABLE II

ATTENUATIONS OF THE INTERFERING SIGNALS COMPARED TO THE DESIRED SIGNAL AT DIFFERENT FREQUENCIES.

\begin{tabular}{|c|c|c|}
\hline Frequency & $\begin{array}{c}\text { Attenuation for } \\
\text { Interference 1 } \\
(\mathrm{dB})\end{array}$ & $\begin{array}{c}\text { Attenuation for } \\
\text { Interference 2 } \\
(\mathrm{dB})\end{array}$ \\
\hline Center frequency, $4.5 \mathrm{GHz}$ & 27.1 & 18.2 \\
Lowest frequency, $4 \mathrm{GHz}$ & 23.9 & 20.5 \\
Highest frequency, 5 GHz & 17.1 & 15.6 \\
\hline
\end{tabular}

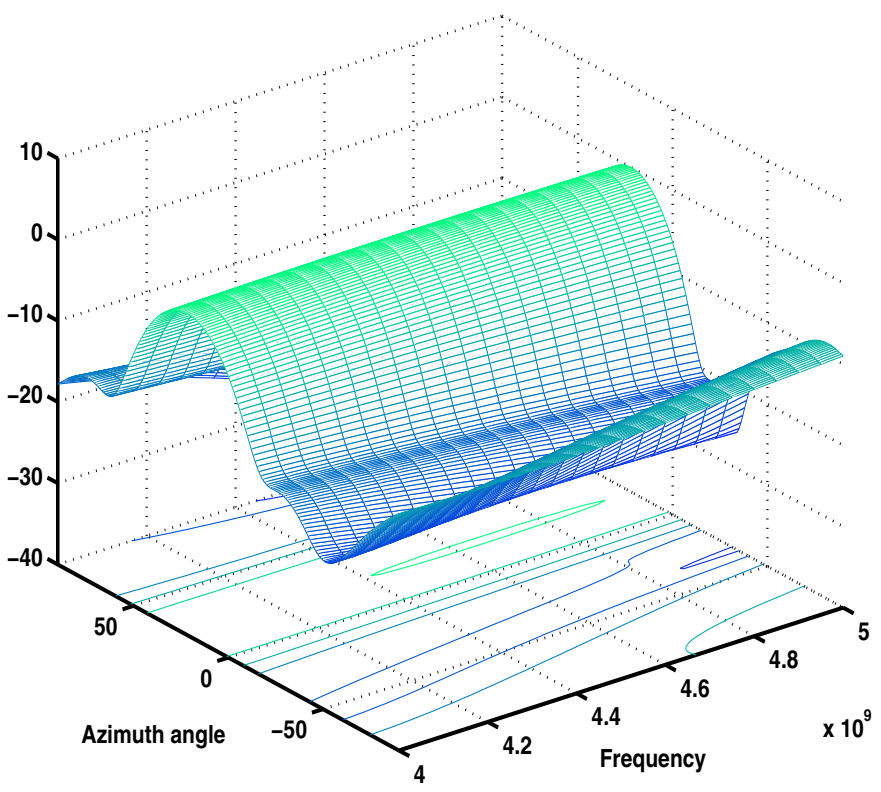

Fig. 5. Frequency variations (in $\mathrm{Hz}$ ) of the beam pattern (in $\mathrm{dB}$ ) with respect to the azimuth angle $\theta$ when the elevation angle is kept constant at the desired value of $\phi_{0}=70^{\circ}$.

to the elevation angle $\phi$ when the azimuth angle is kept at the desired value of $\theta_{0}=20^{\circ}$. Both figures 5 and 6 clearly demonstrate an almost perfect frequency independence for the entire considered frequency range of 4-5 GHz.

\section{CONCLUSION}

An three dimensional adaptive spatially processed wideband conical antenna array is presented. A major advantage of the proposed configuration is the frequency invariance of the directional patterns of the array within a relatively large fractional bandwidth which makes this array a potential candidate for wideband and UWB technologies.

Moreover, unlike conventional wideband antenna arrays which use delay lines or time-domain filters, the proposed system in this paper entirely relies on the spatial processing of the incoming signals without any phase shifter, filter or pure adjustable delay lines. The conical shape of the array structure

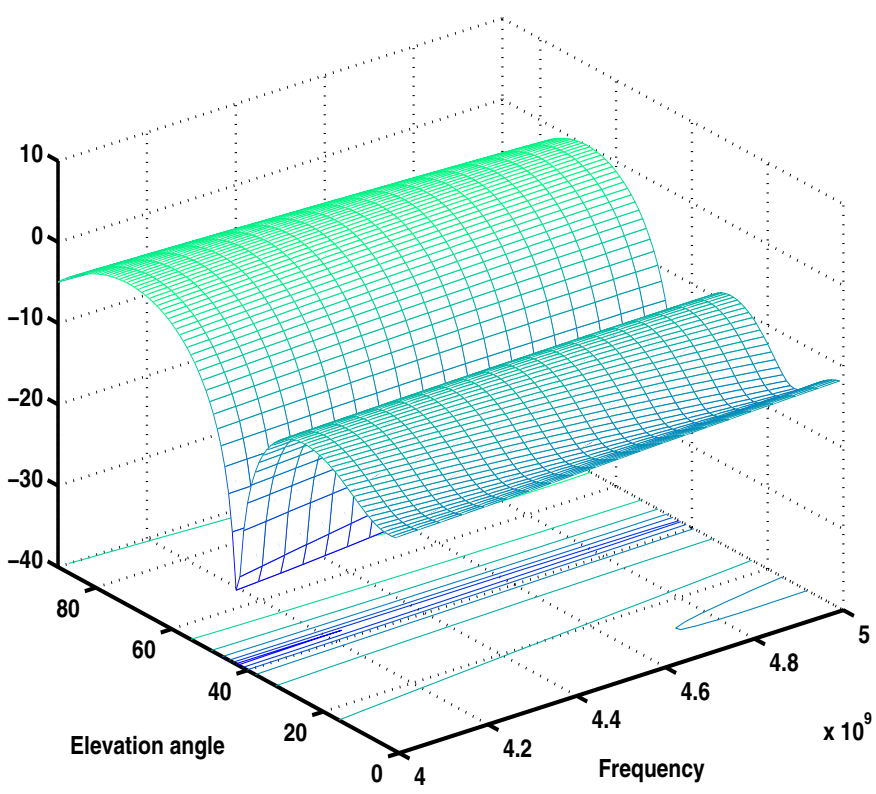

Fig. 6. Frequency variations (in $\mathrm{Hz}$ ) of the beam pattern (in $\mathrm{dB}$ ) with respect to the elevation angle $\phi$ when the azimuth angle is kept constant at the desired value of $\theta_{0}=20^{\circ}$

provides a uniform resolution for all azimuth angles. Finally, the three dimensional conical placement of the array elements provides the possibility of beamformimg in both azimuth and elevation angles.

\section{REFERENCES}

[1] M. Ghavami, "An adaptive wideband array using a single real multiplier for each antenna element", in Proceedings of the International Symposium on Personal, Indoor and Mobile Radio Communications PIMRC'2002, Lisbon, Portugal, September 2002, v. 4, pp. 1805-1809.

[2] M. B. Hawes and W. Liu, "Sparse wideband array design with reweighted iterative optimisation and frequency invariant response", in Proceedings International Conference on Digital Signal Processing, Hong Kong, China, August 2014.

[3] M. Ghavami and R. Kohno, "Recursive fan filters for broadband partially adaptive antenna", IEEE Trans. Communications, 48(2), 2000, pp. 185188

[4] M. Ghavami, "Wideband smart antenna theory using rectangular array structures", IEEE Transactions on Signal Processing, 50(9), 2002, pp. 2143-2150.

[5] S. C. Chan and H. H. Chan, "Uniform concentric circular arrays with frequency-invariant characteristics theory, design, adaptive beamforming and DOA estimation”, IEEE Trans. Signal Processing, 55(1), 2007, pp. 165-177.

[6] D. S. Varma, K. R. Subhashini and G. Lalitha, "Design optimization analysis for multi constraint adaptive antenna array using harmony search and differential evolution techniques", in Proceedings IEEE Conference on Information \& Communication Technologies, Tamil Nadu, India, April 2013.

[7] B. Allen and M. Ghavami, Adaptive Array Systems: Fundamentals and Applications, John Wiley \& Sons, 2005.

[8] B. Widrow and S. D. Stearns, Adaptive Signal Processing, Prentice-Hall, Englewood Cliffs, N.J., 1985. 